\title{
How do autoreactive B cells survive in SLE-autophagy?
}

Data now published in Annals of the

Rheumatic Diseases indicate "autophagy is abnormal in SLE in both T-cell and B-cell compartments, but is more significantly different in B cells," says the lead researcher of the study, Timothy Vyse of King's College London. The researchers found that autophagy is required for differentiation of plasmablasts and that more autophagosomes are formed in naive B cells from patients with systemic lupus erythematosus (SLE) and in a mouse model of SLE, compared with healthy individuals and control mice, respectively. Although autoreactive B cells

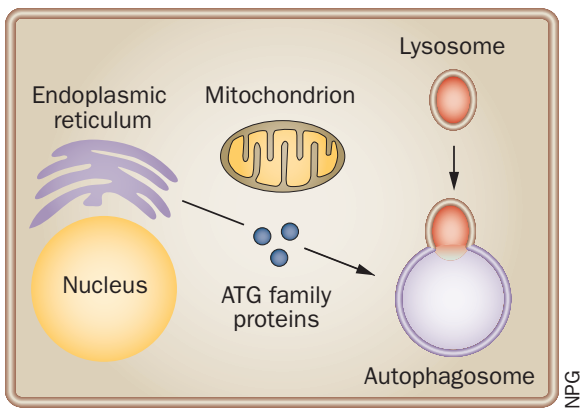

are characteristic of SLE, a role for B-cell autophagy in the pathogenesis of SLE had not previously been studied.

Autophagy is an ATG-family-proteinregulated intracellular mechanism for the vesicular catabolism and recycling of harmful or superfluous cell contents and, therefore, its regulation is important for cell survival and apoptosis. Programmed cell death of autoreactive cells at early stages of ontogeny prevents autoimmunity, indicating that autophagy might be involved in the control of tolerance checkpoints that prevent autoreactive B-cell and T-cell survival.

The researchers found that B-cell autophagy was activated in the early stages of disease in an SLE mouse model (NZB/W F1 mice) and also in patients with SLE. Patients with SLE were excluded from the study if they were being, or had been, treated with rituximab ( $\leq 1$ year) or methylprednisolone ( $\leq 1$ month). Using multispectral-imaging flow cytometry and a tracer dye (CytoID, Enzo Life Sciences) specific for an ATG family member (LC3) the researchers found that, compared with healthy individuals, B cells from patients with SLE $(n=43)$ had more autophagosomes; disease activity (SELENA-SLEDAI) correlated positively with autophagosome density.

Many pharmaceuticals inhibit autophagy and could, therefore, be used as novel treatments for SLE. One difficulty in translating these exciting new findings to the bedside, however, is that autophagy is a physiological mechanism required for the viability and proliferation of all cells, not just autoreactive cells. Vyse speculates "the potential therapeutic role for inhibition of autophagy in lymphocytes in SLE and other autoimmune diseases should be explored," but he agrees that targeting autoreactive cells would be preferential.

Nicholas J. Bernard

Original article Clarke A. J. et al. Autophagy is activated
in systemic lupus erythematosus and required for
plasmablast development. Ann. Rheum. Dis. doi:10.1136/
annrheumdis-2013-204343

